\title{
GENERALIZED STABILITY OF MOTION OF IMPULSIVE LURIE-POSTNIKOV SYSTEMS WITH STRUCTURAL PERTURBATION
}

\author{
A.A. MARTYNYUK ${ }^{1}$ \\ National Ukrainian Academy of Sciences \\ Institute of Mathematics \\ 252057 Kiev-57, Ukraine \\ I.P. STAVROULAKIS \\ University of Ioannina \\ Department of Mathematics \\ 45110 Ioannina, Greece
}

(Received February, 1997; Revised November, 1997)

\begin{abstract}
This paper investigates the absolute stability on $g_{s}$ of the zero solution of Lurie-Postnikov systems with impulses and structural perturbation. A number of absolutely stable on $\mathcal{G}_{s}$ theorems of the Lyapunov type for Lurie-Postnikov systems are proved, extending and generalizing previous work on the subject. These results are applied to some fourth-order LuriePostnikov type systems decomposed into two systems.
\end{abstract} tion.

Key words: Absolute Stability on $\mathfrak{G}_{s}$, Matrix-Valued Lyapunov Func-

AMS subject classifications: $34 \mathrm{D} 10,34 \mathrm{D} 20$.

\section{Introduction}

A system of the Lurie-Postnikov type can be considered complex for the great number of its nonlinearities and/or for the nonstationarity and rich structure of its form.

A system can be considered large scale due to several intrinsic features, among which are included:

(a) high dimensionality,

(b) manifold of the system structure (networks, trees, hierarchical structure, etc.),

\footnotetext{
${ }^{1}$ This work was done when the author was visiting the Department of Mathematics, University of Ioannina, in the framework of the NATO Science Fellowships Programme through the Greek Ministry of National Economy.
} 
(c) multiple connections of the system elements (sub-systems, interconnection in one level and between different levels of hierarchy),

(d) manifold of the elements nature (machines, automata, robots, people-operators),

(e) recurrence of change of the system composition and state (variability of the system's structure, connections and composition),

$(f)$ multiple criteria of the system (difference between local criteria for sub-systems and global criteria for a system in the whole, i.e., their inconsistency).

A direct analysis of a dynamic property (such as stability, controllability, observability, optimality and robustness) of large-scale Lurie-Postnikov systems can be cumbersome, or even impossible. In the framework of stability analysis, this means that the direct Lyapunov method, the most general and powerful for stability analysis, cannot be effectively applied to large-scale Lurie-Postnikov systems, due to the lack of an algorithm for the construction of a set Lyapunov function. This problem can be somewhat diminished by the application of matrix-valued Lyapunov functions which admit a broader class of auxiliary functions suitable for the investigation of stability problems.

The aim of this paper is to apply some general results from [6-9] to the stability analysis of large-scale Lurie-Postnikov impulsive systems under structural perturbation. Sufficient conditions for absolute stability on $\mathcal{G}_{s}$ are established.

\section{Large-Scale Impulsive Lurie-Postnikov System}

We consider the large-scale impulsive system

$$
\begin{gathered}
\frac{d x_{i}}{d t}=\sum_{\ell=1}^{s} S_{i \ell}^{(1)} A_{i \ell} x_{\ell}+\sum_{\ell=1}^{s} S_{i \ell}^{(2)} q_{i \ell} f_{i \ell}\left(\sigma_{i \ell}\right), \\
\sigma_{i \ell}=c_{i \ell}^{T} x, \quad i=1,2, \ldots, s, \quad t \neq \tau_{k}(x), \quad k=1,2, \ldots \\
\Delta x_{i}=\sum_{\ell=1}^{s} J_{k i \ell} x_{\ell}+\sum_{\ell=1}^{s} b_{i \ell} g_{i \ell}\left(\sigma_{i \ell}^{*}\right), \\
\sigma_{i \ell}^{*}=c_{i \ell}^{T} x\left(x_{\tau}(x)\right), \quad i=1,2, \ldots, s, \quad t=\tau_{k}(x), \quad k=1,2, \ldots
\end{gathered}
$$

where

$$
\begin{gathered}
\sigma_{i \ell}^{-1} f_{i \ell}\left(\sigma_{i \ell}\right) \in\left[0, K_{i \ell}\right] \subseteq R_{+}, \\
\left(\sigma_{i \ell}^{*}\right)^{-1} g_{i \ell}\left(\sigma_{i \ell}^{*}\right) \in\left[0, \widetilde{K}_{i \ell}\right] \subseteq R_{+},
\end{gathered}
$$

$A_{i \ell}, J_{k i \ell}$ are constant matrices, $x_{i} \in R^{n_{i}}, n_{1}+n_{2}+\ldots+n_{s}=n, g_{i \ell}, b_{i \ell}$, are constant vectors and $K_{i \ell}, \widetilde{K}_{i \ell}$ are positive constants, all of the appropriate dimensions. The matrices $S_{i \ell}^{(1)}, S_{i \ell}^{(2)}$ and the structural set $g_{s}$ are described in Appendix 1. The independent subsystems corresponding to system (1) are obtained by replacing $x$ in (1) with $x^{i}$, where $x^{i}=\left(0, \ldots, 0, x_{i}^{T}, 0, \ldots, 0\right)^{T} \in R^{n_{i}}$ : 


$$
\begin{aligned}
& \frac{d x_{i}}{d t}=A_{i i} x_{i}+q_{i i} f_{i i}\left(\tilde{\sigma}_{i i}\right), \quad t \neq \tau_{k}\left(x^{i}\right) \\
& \Delta x_{i}=J_{k i i} x_{i}+b_{i i} g_{i i}\left(\tilde{\sigma}_{i i}^{*}\right), \quad t=\tau_{k}\left(x^{i}\right),
\end{aligned}
$$

where

$$
\tilde{\sigma}_{i i}=c_{i i}^{T} x_{i}, \quad \tilde{\sigma}_{i i}^{*}=\tilde{c}_{i i}^{T} x_{i}\left(\tau_{k}\left(x^{i}\right)\right), \quad i=1,2, \ldots, s .
$$

In order to simplify system (1), we introduce the designations

$$
\begin{gathered}
f_{i}\left(x^{i}\right)=A_{i i} x_{i}+q_{i i} f_{i i}\left(\tilde{\sigma}_{i i}\right), \tilde{\sigma}_{i i}=c_{i i}^{T} x_{i}, t \neq \tau_{k}\left(x^{i}\right), k=1,2, \ldots \\
F_{i}(x, S)=\sum_{\substack{\ell=1 \\
\ell \neq i}}^{s} S_{i \ell}^{(1)} A_{i \ell} x_{\ell}+\sum_{\substack{\ell=1 \\
\ell \neq i}}^{s} S_{i \ell}^{(2)} q_{i \ell} f_{i \ell}\left(\sigma_{i \ell}\right) \\
+S_{i i}^{(2)} q_{i i}\left[f_{i i}\left(\sigma_{i i}\right)-f_{i i}\left(\tilde{\sigma}_{i i}\right)\right], \sigma_{i \ell}=c_{i \ell}^{T} x_{i}, \quad t \neq \tau_{k}\left(x^{i}\right), k=1,2, \ldots ; \\
g_{i}\left(x^{i}\right)=J_{k i i} x_{i}+b_{i i} g_{i i}\left(\tilde{\sigma}_{i i}^{*}\right), \quad t=\tau_{k}\left(x^{i}\right), k=1,2, \ldots \\
G_{i}(x)=\sum_{\substack{\ell=1 \\
\ell \neq i}}^{s} J_{k i \ell} x_{\ell}+\sum_{\substack{\ell=1 \\
\ell \neq i}}^{s} b_{i \ell} g_{i \ell}\left(\sigma_{i \ell}^{*}\right) \\
+b_{i i}\left[g_{i i}\left(\tilde{\sigma}_{i i}^{*}\right)-g_{i i}\left(\tilde{\sigma}_{i i}^{*}\right)\right], \quad t=\tau_{k}(x), k=1,2, \ldots .
\end{gathered}
$$

Then system (1) becomes

$$
\begin{gathered}
\frac{d x_{i}}{d t}=f_{i}\left(x^{i}\right)+F_{i}(x, S), \quad t=\tau_{k}(x), \quad S \in \mathcal{G}_{s} \quad i=1,2, \ldots, s \quad k=1,2, \ldots \\
\Delta x_{i}=g_{i}\left(x^{i}\right)+G_{i}(x), t=\tau_{k}(x), k=1,2, \ldots \quad i=1,2, \ldots, s .
\end{gathered}
$$

\section{Matrix-Valued Lyapunov Function}

Together with system (1) and subsystems (2) we consider the matrix-valued function

$$
U(x)=\left[u_{i j}\left(x_{i}, x_{j}\right)\right], u_{i j}=u_{j i}, \quad i=j=1,2, \ldots, s
$$

the elements of which are determined as

$$
u_{i j}\left(x_{i}, x_{j}\right)=x_{i}^{T} P_{i j} x_{j}, \quad i, j=1,2, \ldots, s
$$

where $x_{i} \in R^{n_{i}}, x_{j} \in R^{n_{j}}, P_{i i}$ are symmetric, positive definite matrices, and $P_{i j}$ are constant matrices for all $i, j=1,2, \ldots, s$.

It is known (see $[1,3]$ ) that the functions (5) satisfy the estimates

$$
\lambda_{m}\left(P_{i i}\right)\left\|x_{i}\right\|^{2} \leq u_{i i}\left(x_{i}\right) \leq \lambda_{M}\left(P_{i i}\right)\left\|x_{i}\right\|^{2},
$$


(b)

$$
\begin{gathered}
\forall x_{i} \in R^{n_{i}}, \quad i=1,2, \ldots, s \\
-\lambda_{M}^{1 / 2}\left(P_{i j} P_{i j}^{T}\right)\left\|x_{i}\right\|\left\|x_{j}\right\| \leq u_{i j}\left(x_{i}, x_{j}\right) \leq \lambda_{M}^{1 / 2}\left(P_{i j} P_{i j}^{T}\right)\left\|x_{i}\right\|\left\|x_{j}\right\| \\
\forall x_{i} \in R^{n_{i}}, x_{j} \in R^{n_{j}}, \quad \forall(i \neq j)=1,2, \ldots, s
\end{gathered}
$$

where $\lambda_{m}\left(P_{i i}\right)$ are the minimal and $\lambda_{M}\left(P_{i i}\right)$ are the maximal eigenvalues of the matrices $P_{i i}$, and $\lambda_{M}^{1 / 2}\left(P_{i j} P_{i j}^{T}\right)$ is the norm of the matrices $P_{i j}$.

Using the matrix-valued function (4) and the constant vector $\eta=(1,1, \ldots, 1) \in$ $R_{+}^{s}$, we construct the function

$$
V(x, \eta)=\eta^{T} U(x) \eta
$$

and consider its total derivative

where

$$
D V(x, \eta)=\eta^{T} D U(x) \eta
$$

$$
D U(x)=\left[D u_{i j}\left(x_{i}, x_{j}\right)\right], \quad i, j=1,2, \ldots, s
$$

along the solutions of system (1).

Lemma 1: If the estimates of (6) are satisfied, then for the function (7) the twosided inequality

$$
u^{T} A u \leq V(x, \eta) \leq u^{T} B u \quad \forall x \in R^{n}
$$

holds true, where

$$
\begin{gathered}
u^{T}=\left(\left\|x_{1}\right\|,\left\|x_{2}\right\|, \ldots,\left\|x_{s}\right\|\right), \\
A=\left[\bar{\alpha}_{i j}\right], \quad B=\bar{\alpha}_{i j}, \quad i, j=1,2, \ldots, s \\
\underline{\alpha}_{i i}=\lambda_{m}\left(P_{i i}\right), \quad \bar{\alpha}_{i i}=\lambda_{M}\left(P_{i i}\right) \\
\underline{\alpha}_{i j}=\underline{\alpha}_{j i}=-\bar{\alpha}_{i j}=-\bar{\alpha}_{j i}=-\lambda_{M}^{1 / 2}\left(P_{i j} P_{i j}^{T}\right) .
\end{gathered}
$$

Proof: The proof of Lemma 1 follows from Lemma 1 in [7].

Corollary 1: If inequality (9) is satisfied, then

and for

$$
\begin{gathered}
\lambda_{m}(A)\|u\|^{2} \leq V(x, \eta) \leq \lambda_{M}(B)\|u\|^{2} \\
\forall x \in R^{n} \quad \eta=(1,1, \ldots, \lambda) \in R_{+}^{s}
\end{gathered}
$$

$$
\begin{gathered}
\lambda_{m}(A)>0, \quad \lambda_{M}(B)>0 \\
\lambda_{M}^{-1}(B) V(x, \eta) \leq\|u\|^{2} \leq \lambda_{m}^{-1}(A) V(x, \eta) .
\end{gathered}
$$

Lemma 2: If for system (1) the matrix-valued function (4) is constructed with the elements (5), then for the derivatives of functions (5) along with solutions of (1) 
for $t \neq \tau_{k}(x), k=1,2, \ldots$ the estimates

(a)

$$
\begin{gathered}
\left(D x_{i} U_{i i}\right)^{T} f_{i}\left(x^{i}\right) \leq \rho_{i}^{(1)}\left\|x_{i}\right\|^{2} \forall x_{i} \in R^{n_{i}}, \quad i=1,2, \ldots, s \\
\sum_{i=1}^{s}\left(D x_{i} U_{i i}\right)^{T} F_{i}(x, S)+2 \sum_{i=1}^{s} \sum_{\substack{j=2 \\
j>i}}^{s} \\
\times\left\{\left(D x_{i} u_{i j}\right)^{T}\left(f_{i}\left(x^{i}\right)+F_{i}(x, S)\right)+\left(D x_{j} u_{i j}\right)^{T}\left(f_{i}\left(x^{i}\right)+F_{j}(x, S)\right)\right\} \\
\leq \sum_{i=1}^{S} \rho_{i}^{(2)}(S)\left\|x_{i}\right\|^{2}+2 \sum_{i=1}^{s} \sum_{\substack{j=2 \\
j>i}}^{s} \rho_{i j}(S)\left\|x_{i}\right\|\left\|x_{j}\right\| \\
\forall\left(x_{i}, x_{j}\right) \in R^{n_{i} \times R^{n}, \quad \forall s \in \mathcal{G}_{s},}
\end{gathered}
$$

(b)

are satisfied, where $\rho_{i}^{(1)}$ and $\rho_{i}^{(2)}(S), i=1,2, \ldots, s$ are maximal eigenvalues of the matrices

$$
\begin{gathered}
P_{i i} A_{i i}+A_{i i}^{T} D_{i i}+P_{i i} q_{i i} k_{i i}^{*}\left(c_{i i}^{i}\right)^{T}+\left(q_{i i} k_{i i}^{*}\left(c_{i i}^{i}\right)^{T}\right)^{T} P_{i i} \\
\sum_{\ell=1}^{i-1}\left\{\left[\left(S_{\ell_{i}}^{(1)} A_{\ell_{i}}\right)^{T}+\left(S_{\ell i}^{(t)} q_{\ell_{i}} k_{\ell_{i}}^{*}\left(c_{\ell_{i}}^{i}\right)^{T}\right)^{T}\right] P_{\ell i}\right. \\
\left.+P_{\ell i}^{T}\left[S_{\ell_{i}}^{(1)} A_{\ell_{i}}+S_{\ell_{i}}^{(2)} q_{\ell_{i}} k_{\ell_{i}}^{*}\left(c_{\ell_{i}}^{i}\right)^{T}\right]\right\} \\
+\sum_{\ell=i+1}^{s}\left\{P_{i \ell}\left[S_{\ell_{i}}^{(1)} A_{\ell_{i}}+S_{\ell_{i}}^{(2)} q_{\ell_{i}} k_{\ell_{i}}^{*}\left(c_{\ell_{i}}^{i}\right)^{T}\right]\right. \\
\left.+\left[\left(S_{\ell_{i}}^{(1)} A_{\ell_{i}}\right)^{T}+\left(S_{\ell_{i}}^{(2)} q_{\ell_{i}} k_{\ell_{i}}^{*}\left(c_{\ell_{i}}^{i}\right)^{T}\right)^{T}\right] P_{i \ell}^{T}\right\} \\
+\sum_{j=2}^{s}\left\{P_{i j} S_{j i}^{(2)} q_{j i} k_{j i}^{* *}\left(c_{j i}^{i}\right)^{T}+\left(S_{j i}^{(2)} q_{j i} k_{j i}^{* *}\left(c_{j i}^{i}\right)^{T}\right)^{T} P_{i j}\right\}
\end{gathered}
$$

respectively, $\rho_{i j}(S), i<j, i=1,2, \ldots, s, j=2, \ldots, s$ are the norms of the matrices

$$
\begin{gathered}
\sum_{\ell=1}^{j-1}\left[\left(S_{\ell_{i}}^{(1)} A_{\ell_{i}}\right)^{T}+\left(S_{\ell_{i}}^{(2)} q_{\ell_{i}} k_{\ell_{i}}^{*}\left(c_{\ell_{i}}^{i}\right)^{T}\right] P_{\ell j}\right. \\
+\sum_{\ell=j+1}^{s}\left[\left(S_{\ell_{i}}^{(1)} A_{\ell_{i}}\right)^{T}+\left(S_{\ell_{i}}^{(2)} q_{\ell_{i}} k_{\ell_{i}}^{*}\left(c_{\ell_{i}}^{i}\right)^{T}\right)^{T}\right] P_{\ell_{j}} \\
+\sum_{\ell=1}^{j-1} P_{\ell_{i}}^{T}\left[S_{\ell_{j}}^{(1)} A_{\ell_{j}}+S_{\ell_{j}}^{(2)} q_{\ell_{j}} k_{\ell_{j}}^{*}\left(c_{\ell_{j}}^{j}\right)^{T}\right]
\end{gathered}
$$




$$
\begin{gathered}
+\sum_{\ell=i+1}^{s} P_{i \ell}\left[S_{\ell j}^{(1)} A_{\ell j}+S_{\ell j}^{(2)} q_{\ell j} k_{\ell j}^{*}\left(c_{\ell j}^{j}\right)^{T}\right] \\
+\frac{1}{2}\left\{P_{i i}\left(S_{i j}^{(1)} A_{i j}\right)+\left(S_{i j}^{(1)} A_{i j}\right)^{T} P_{i i}+P_{i i}\left(S_{i j}^{(2)} q_{i j} k_{i j}^{*}\left(c_{i j}^{j}\right)^{T}\right)\right. \\
\left.+\left(S_{i j}^{(2)} q_{i j} k_{i j}^{*}\left(c_{i j}^{j}\right)^{T}\right) P_{i i}+P_{i i}\left(q_{i i} k_{i i}^{*}\left(c_{i i}^{j}\right)^{T}\right)+\left(q_{i i} k_{i j}^{*}\left(c_{i i}^{j}\right)^{T}\right)^{T} P_{i i}\right\} \\
+\frac{1}{2}\left\{P_{j i}\left(S_{j i}^{(1)} A_{j i}\right)+\left(S_{j i}^{(1)} A_{j i}\right)^{T} P_{j i}+P_{j j}\left(S_{j i}^{(2)} q_{j i} k_{j i}^{*}\left(c_{j i}^{i}\right)^{T}\right)\right. \\
\left.+\left(S_{j i}^{(2)} q_{j i} k_{j i}^{*}\left(c_{j i}^{i}\right)^{T}\right)^{T} P_{j j}+P_{j j}\left(q_{j j} k_{j j}^{*}\left(c_{j j}^{i}\right)^{T}\right)+\left(q_{j j} k_{j j}^{*}\left(c_{j j}^{i}\right)^{T}\right)^{T} P_{j j}\right\} \\
i=1,2, \ldots, s, \quad j=1,2, \ldots, s
\end{gathered}
$$

respectively.

Here

$$
\begin{aligned}
& k_{j i}^{*}=\left\{\begin{array}{cc}
k_{i j} & \text { for } \sigma_{i j}\left(S_{i j}^{(2)} q_{i j}\right)^{T} P_{i j} x_{j}>0, \quad i, j=1,2, \ldots, s, \\
0 & \text { in other cases }
\end{array}\right.
\end{aligned}
$$

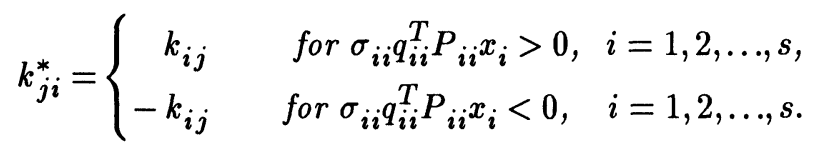

The proof is carried out the same way as in Lemma 2 in [7].

Lemma 3: If all conditions of Lemma 2 are satisfied, then for (8)

where

$$
D V(x, \eta) \leq u^{T} \Theta u, \quad \forall(x, S) \in R^{n} \times \mathrm{g}_{s}
$$

$$
\begin{gathered}
\Theta=\left[\theta_{i j}\right], \quad i, j=1,2, \ldots, s, \quad \theta_{i i}=\rho_{i}^{(1)}+\rho_{i}^{(2)}\left(S^{*}\right), \\
\theta_{i j}=\theta_{j i}=\rho_{i j}\left(S^{*}\right), \quad S^{*} \in \mathcal{G}_{s}, \quad i \neq j, \quad i, j=1,2, \ldots, s
\end{gathered}
$$

is the constant matrix such that

and

$$
\rho_{i}^{(2)}(S) \leq \rho_{i}^{(2)}\left(S^{*}\right)
$$

$$
\rho_{i j}(S)<\rho_{i j}\left(S^{*}\right) \text {. }
$$

The proof of this lemma is similar to that of Lemma 3 in [7].

Corollary 2: If inequalities (10) and (11) are satisfied, then for (12)

$$
D V(x, \eta) \leq \begin{cases}\lambda_{m}(\Theta) \lambda_{M}^{-1}(B) V(x, \eta) & \text { for } \lambda_{M}(\Theta)<0 \\ \lambda_{m}(\Theta) \lambda_{m}^{-1}(A) V(x, \eta) & \text { for } \lambda_{M}(\Theta)>0\end{cases}
$$


The proof follows from Lemma 3 and Corollary 1.

Lemma 4: For the function (7) for $t=\tau_{k}(x), k=1,2, \ldots$ due to system (1), the estimates

hold true, where

$$
\begin{gathered}
V\left(x+J_{k}^{*}(x), \eta\right)-V(x, \eta) \leq u_{k}^{T} \Lambda u_{k} \\
V\left(x+J_{k}^{*}(x), \eta\right) \leq u_{k}^{T} \Lambda^{*} u_{k}
\end{gathered}
$$

$$
\begin{aligned}
& u_{k}^{T}=\left(\left\|x_{1}\left(\tau_{k}(x)\right)\right\|,\left\|x_{2}\left(\tau_{k}(x)\right)\right\|, \ldots,\left\|x_{s}\left(\tau_{k}(x)\right)\right\|\right) \\
& J_{k}^{*}(x)=\sum_{l=1}^{s} J_{k i l} x_{l}+\sum_{l=1}^{s} b_{i l} q_{i l}\left(\sigma_{i l}^{1}\right) \\
& \Lambda=\left[\omega_{i j}\right], \omega_{i j}=\omega_{j i}, \quad i, j=1,2, \ldots, s ; \\
& \Lambda^{*}=\left[\xi_{i j}\right], \quad \xi_{i j}=\xi_{j i}, \quad i, j=1,2, \ldots, s ; \\
& \omega_{i i}=\lambda_{M}\left(\Omega_{i i}\right), \quad \omega_{i j}=\lambda_{M}^{1 / 2}\left(\Omega_{i j} \Omega_{i j}^{T}\right), \quad i \neq j, \quad i, j=1,2, \ldots s ; \\
& \xi_{i i}=\lambda_{M}\left(\Psi_{i i}\right), \quad \xi_{i j}=\lambda_{M}^{1 / 2}\left(\Psi_{i j} \Psi_{i j}^{T}\right), \quad i \neq j, \quad i, j=1,2, \ldots, s ; \\
& \Omega_{i i}=P_{i i} J_{k i i}+J_{k i i}^{T} P_{i i}+\sum_{j=1}^{s} J_{k j i}^{T} P_{j j} J_{k j i}+P_{i i}\left(\sum_{\ell=1}^{s} b_{i \ell} \tilde{k}_{i \ell}^{*}\left(\tilde{c}_{\ell}^{i}\right)^{T}\right) \\
& +\left(\sum_{\ell=1}^{s} b_{i \ell} \tilde{k}_{i \ell}^{*}\left(\tilde{c}_{i \ell}^{i}\right)^{T}\right)^{T} P_{i i}+\sum_{j=1}^{s}\left\{J_{k j i} P_{j j}\left(\sum_{\ell=1}^{s} b_{j \ell} \tilde{k}_{j \ell}^{*}\left(\tilde{c}_{j \ell}^{j}\right)^{T}\right)\right. \\
& \left.+\left(\sum_{\ell=1}^{s} b_{j \ell} \tilde{k}_{j \ell}^{*}\left(\tilde{c}_{j \ell}^{j}\right)^{T}\right)^{T} P_{j j} J_{k j i}\right\} \\
& +\sum_{j=1}^{s}\left(\sum_{\ell=1}^{s} b_{j \ell} \tilde{k}_{j \ell}^{*}\left(\tilde{c}_{j \ell}^{j}\right)^{T}\right)^{T} P_{j j}\left(\sum_{\ell=1}^{s} b_{j \ell} \tilde{k}_{j \ell}^{*}\left(\tilde{c}_{j \ell}^{j}\right)^{T}\right) \\
& +\sum_{j=1}^{s}\left\{P_{i j} J_{k j i}+J_{k j i}^{T} P_{i j}+P_{i j}\left(\sum_{\ell=1}^{s} b{ }_{j \ell} \tilde{k}_{j \ell}^{*}\left(\tilde{c}_{j \ell}^{i}\right)^{T}\right)\right. \\
& +\left(\sum_{\ell=1}^{s} b{ }_{j \ell} \tilde{k}_{j \ell}^{*}\left(\widetilde{c}_{j \ell}^{i}\right)^{T}\right)^{T} P_{i j}+\sum_{\ell=1}^{s}\left(J_{k \ell i}^{T} P_{\ell j} J_{k j i}+J_{k j i}^{T} P_{\ell j} J_{k \ell i}\right) \\
& +J_{k j i}^{T} P_{i j}\left(\sum_{\ell=1}^{s} b_{j \ell} \tilde{k}_{j \ell}^{*}\left(\tilde{c}_{j \ell}^{i}\right)^{T}\right)+\left(\sum_{\ell=1}^{s} b_{j \ell} \tilde{k}_{j \ell}^{*}\left(\tilde{c}_{j \ell}^{i}\right)^{T}\right)^{T} P_{i j} J_{k j i}
\end{aligned}
$$




$$
\begin{aligned}
& +\left(\sum_{\ell=1}^{s} b_{i \ell} \tilde{k}_{i \ell}^{*}\left(\tilde{c}_{i \ell}^{i}\right)^{T}\right)^{T} P_{i j}\left(\sum_{\ell=1}^{s} b_{j \ell} \tilde{k}_{j \ell}^{*}\left(\tilde{c}_{j \ell}^{i}\right)^{T}\right) \\
& \left.+\left(\sum_{\ell=1}^{s} b_{j \ell} \tilde{k}_{j \ell}^{*}\left(\tilde{c}_{j \ell}^{i}\right)^{T}\right)^{T} P_{i j}\left(\sum_{\ell=1}^{s} b_{i \ell} \tilde{k}_{i \ell}^{*}\left(\tilde{c}_{i \ell}^{i}\right)^{T}\right)\right\}, \quad i=1,2, \ldots, s \\
& \Omega_{i j}=P_{i i} J_{k i j}+J_{k j i}^{T} P_{j j}+P_{i i}\left(\sum_{\ell=1}^{s} b_{i \ell} \tilde{k}_{i \ell}^{*}\left(\tilde{c}_{i \ell}^{i}\right)^{T}\right) \\
& +\left(\sum_{\ell=1}^{s} b_{j \ell} \tilde{k}_{j \ell}^{*}\left(\tilde{c}_{j \ell}^{j}\right)^{T}\right)^{T} P_{j j}+\sum_{\ell=1}^{s}\left(J_{k \ell i}^{T} P_{\ell \ell} J_{k \ell j}+J_{k \ell j}^{T} P_{\ell \ell} J_{k \ell i}\right) \\
& \sum_{r=1}^{s}\left\{J_{k r i}^{T} P_{r r}\left(\sum_{\ell=1}^{s} b_{r \ell} \tilde{k}_{r \ell}^{*}\left(\tilde{c}_{r \ell}^{r}\right)^{T}\right)+\left(\sum_{\ell=1}^{s} b_{r \ell} \tilde{k}_{r \ell}^{*}\left(\tilde{c}_{r \ell}^{r}\right)^{T}\right)^{T} P_{r r} J_{k r i}\right\} \\
& +\left(\sum_{\ell=1}^{s}\left(P_{i \ell} J_{k \ell j}+J_{k \ell j}^{T} B_{\ell j}\right)\right)+P_{i j}\left(\sum_{\ell=1}^{s} b_{j \ell} \tilde{k}_{j \ell}^{*}\left(\tilde{c}_{j \ell}^{j}\right)^{T}\right) \\
& +\left(\sum_{\ell=1}^{s} b_{i \ell} \tilde{k}_{i \ell}^{*}\left(\tilde{c}_{i \ell}^{i}\right)^{T}\right)^{T} P_{i i}+\left(\sum_{\ell=1}^{s} b_{i \ell} \tilde{k}_{i \ell}^{*}\left(\tilde{c}_{i \ell}^{j}\right)^{T}\right) \\
& +\left(\sum_{\ell=1}^{s} b_{i \ell} \tilde{k}_{i \ell}^{*}\left(\tilde{c}_{i \ell}^{i}\right)^{T}\right) P_{i j}+\sum_{\ell=1}^{s} \sum_{\substack{r=1 \\
r \neq \ell}}^{s} J_{k \ell j}^{T} P_{\ell r} J_{k r j}^{T} \\
& +\sum_{\substack{r \neq 1 \\
r \neq i, j}}^{s}\left\{J_{k r j}^{T} P_{r j}\left(\sum_{\ell=1}^{s} b_{j \ell} \tilde{k}_{j \ell}^{*}\left(\tilde{c}_{j \ell}^{j}\right)^{T}\right)^{T}+\left(\sum_{\ell=1}^{s} b_{i \ell} \tilde{k}_{i \ell}^{*}\left(\tilde{c}_{i \ell}^{i}\right)^{T}\right)^{T} P_{i r} J_{k r j}\right\} \\
& +\left(\sum_{\ell=1}^{s} b_{i \ell} \tilde{k}_{i \ell}^{*}\left(\tilde{c}_{i \ell}^{i}\right)^{T}\right)^{T} P_{i j}\left(\sum_{\ell=1}^{s} b_{j \ell} \tilde{k}_{j \ell}^{*}\left(\tilde{c}_{j \ell}^{j}\right)^{T}\right), i \neq j, i, j=1,2, \ldots, s ; \\
& \Psi_{i i}=P_{i i}+\Omega_{i i}, \quad \Psi_{i j}=P_{i j}+\Omega_{i j}, \quad i \neq j=1,2, \ldots, s .
\end{aligned}
$$

Here

$$
\tilde{k}_{i j}^{*}=\left\{\begin{array}{cc}
\tilde{k}_{i j} \text { if the corresponding multiplier is positive; } \\
0 & \text { in other cases. }
\end{array}\right.
$$

The proof is similar to that of Lemma 4 in [7].

Corollary 3: Under all conditions of Lemma 4 for function (7) when $t=\tau_{k}(x)$, 
$k=1,2, \ldots$ the estimates

$$
V\left(x+J_{k}(x), \eta\right)-V(x, \eta) \leq \gamma V(x, \eta)
$$

where

and

$$
\gamma= \begin{cases}\lambda_{M}(\Lambda) \lambda_{M}^{-1}(B) & \text { for } \lambda_{M}(\Lambda)<0 \\ \lambda_{M}(\Lambda) \lambda_{m}^{-1}(A) & \text { for } \lambda_{M}(\Lambda)>0\end{cases}
$$

$$
V\left(x+J_{k}(x), \eta\right) \leq \gamma^{*} V(x, \eta)
$$

where

hold true.

$$
\gamma^{*}= \begin{cases}\lambda_{M}\left(\Lambda^{*}\right) \lambda_{M}^{-1}(B) & \text { for } \lambda_{M}\left(\Lambda^{*}\right)<0 \\ \lambda_{M}\left(\Lambda^{*}\right) \lambda_{m}^{-1}(A) & \text { for } \lambda_{M}\left(\Lambda^{*}\right)>0\end{cases}
$$

The proof follows from Lemma 4 and Corollary 1.

\section{Absolute Stability of Impulsive Lurie-Postnikov Systems}

For system (1), the following stability problem is formulated. It is necessary to formulate conditions related to the coefficients which appear in the system and also to introduce structural perturbation, such that the trivial solution of system (1) is asymptotically stable in the whole on $\mathcal{G}_{s}$ for an arbitrary function $f$ of the class under consideration.

In view of the results from [2,6-9] we shall introduce the following notions.

Definition 1: The zero solution $x=0$ of (1) is absolutely stable under structural perturbation (i.e., absolutely stable on $\mathfrak{g}_{s}$ ) if it is absolutely stable for each $S \in \mathfrak{g}_{s}$ in the sense of Lurie-Postnikov [5].

The above lemmas and corollaries allow us to establish sufficient conditions for absolute stability of the zero solution of system (1) on $\mathfrak{G}_{s}$.

Theorem 1: Let system (1) be such that the matrix-valued function (4) is constructed with the elements (5) and

(i) the matrix $A$ in (9) is positive definite, i.e., $\lambda_{m}(A)>0$;

(ii) the matrix $\Theta$ in (12) is negative semidefinite or equals to zero, i.e., $\lambda_{M}(\Theta) \leq 0$;

(iii) the matrix $\Lambda$ in (14) is negative definite.

Then the zero solution of system (1) is absolutely stable on $\mathfrak{G}_{s}$.

Proof: Under all conditions of Theorem 1,

(a) the function $V(x, \eta)$ (see (7)) is positive definite;

(b) for the function $V(x, \eta)$ and $t \neq \tau_{k}(x), k=1,2, \ldots$ we have

$$
D V(x, \eta) \leq 0 \quad \forall S \in \mathcal{G}_{s}, \text { and } x \in R^{n} ;
$$

(c) for the function $V(x, \eta)$ and $t=\tau_{k}(x), k=1,2, \ldots$ we have

$$
V\left(x+J_{k}(x), \eta\right)-V(x, \eta) \leq \lambda_{M}(\Lambda) \lambda_{m}^{-1}(B) V(x, \eta) \quad \forall x \in R^{n} .
$$


By Theorem 1 from [7] for $(a)-(c)$ the zero solution of system (1) is asymptotically stable in the whole on $\mathcal{G}_{s}$. Since here $\mathcal{N}_{i x}=R^{n i}, i=1,2, \ldots, s$ and $\mathcal{N}_{x}=\mathcal{N}_{1 x} \times \ldots$ $\times \mathcal{N}_{s x}=R^{n}$.

Theorem 2: Let system (1) be such that the matrix-valued function (4) is constructed with the elements (5) and

(i) the matrix $A$ in (9) is positive definite, i.e., $\lambda_{m}(A)>0$;

(ii) the matrix $\Theta$ in (12) is negative definite, i.e., $\lambda_{M}(\Theta)<0$;

(iii) the matrix $\Lambda^{*}$ in (15) is positive definite, i.e., $\lambda_{M}\left(\Lambda^{*}\right)>0$;

(iv) the function $\tau_{k}(x), k=1,2, \ldots$, satisfy the inequality

$$
\sup _{k}\left(\min _{x \in R^{n}} \tau_{k+1}(x)-\max _{x \in R^{n}} \tau_{k}(x)\right)=\theta>0 .
$$

If for some $\gamma>0$, the inequality

$$
-\frac{\lambda_{M}(B)}{\lambda_{M}(\Theta)} \ln \frac{\lambda_{M}\left(\Lambda^{*}\right)}{\lambda_{m}(A)} \leq \theta-\gamma
$$

is satisfied, then the zero solution of system (1) is absolutely stable on $\mathrm{g}_{\boldsymbol{s}}$.

The proof follows from Lemmas 1-4 and Theorem 2 in [7].

Theorem 3: Let system (1) be such that the matrix-valued function (4) is constructed with the elements (5) and

(i) the matrix $A$ in (9) is positive definite, i.e., $\lambda_{m}(A)>0$;

(ii) the matrix $\Theta$ in (12) is positive definite, i.e., $\lambda_{M}(\Theta)>0$;

(iii) the matrix $\Lambda^{*}$ in (15) is positive definite, i.e., $\lambda_{M}\left(\Lambda^{*}\right)>0$;

(iv) the functions $\tau_{k}(x), k=1,2, \ldots$, for some $\theta_{1}>0$ satisfy the inequality

$$
\max _{x \in R^{n}} \tau_{k}(x)-\min _{x \in R^{n}} \tau_{k-1}(x) \leq \theta_{1}, \quad k=1,2, \ldots
$$

If for some $\gamma>0$ the inequality

$$
\frac{\lambda_{m}(A)}{\lambda_{M}(\Theta)} \ln \frac{\lambda_{m}(A)}{\lambda_{m}\left(\Lambda^{*}\right)} \geq \theta_{1}+\gamma
$$

is satisfied, then the zero solution of system (1) is absolutely stable on $\mathrm{g}_{\boldsymbol{s}}$.

Proof: The statement of Theorem 3 follows from Lemmas 1-4 and Theorem 3 in [7].

Example: Let system (1) be a fourth-order system of the Lurie-Postnikov type decomposed into two subsystems determined by the following vectors and matrices:

$$
\begin{gathered}
A_{i i}=\left(\begin{array}{cc}
-4 & 1 \\
1 & -4
\end{array}\right), i=1,2, \\
A_{12}=A_{21}=\left(\begin{array}{cc}
1 & 0 \\
0 & 1
\end{array}\right), \quad q_{i \ell}=\left(\begin{array}{l}
1 \\
1
\end{array}\right), \\
c_{i \ell}^{r}=\left(\begin{array}{c}
1 \\
0
\end{array}\right), \quad k_{i \ell}=1, \quad i, \ell, r=1,2 ;
\end{gathered}
$$




$$
\begin{gathered}
J_{k i i}=\operatorname{diag}\{-1,1\}, \quad J_{k 12}=J_{k 21}=\operatorname{diag}\{0,1 ; 0,1\} \\
b_{i \ell}=\left(\begin{array}{cc}
0, & 1 \\
0, & 1
\end{array}\right), \widetilde{e}_{i \ell}^{r}=\left(\begin{array}{cc}
0, & 1 \\
0
\end{array}\right), \tilde{k}_{i \ell}=1, \quad i, \ell, r=1,2 ; \\
S_{i i}^{(r)}=\operatorname{diag}\{1,1\}, \quad S_{i j}^{(r)}=s_{i j}^{(r)} \quad \operatorname{diag}\{1,1\} \\
u \leq s_{i j}^{(r)} \leq 1, \quad i, j, r=1,2, \quad i \neq j .
\end{gathered}
$$

For this example, the elements of the matrix-valued function (4) are taken in the form

$$
\begin{gathered}
u_{i i}\left(x_{i}\right)=x_{i}^{T} I_{2} x_{i}, \quad i=1,2 \\
u_{12}\left(x_{1}, x_{2}\right)=U_{21}\left(x_{1}, x_{2}\right)=x_{1}^{T} 0,1 I_{2} x_{2}
\end{gathered}
$$

where $I_{2}=\operatorname{diag}\{1,1\}$.

Let also $\eta^{T}=(1,1) \in R_{+}^{2}$. It is easy to verify that the matrices

$$
A=\left(\begin{array}{cc}
1 & -0,1 \\
-0,1 & 1
\end{array}\right), B=\left(\begin{array}{cc}
1 & 0,1 \\
0,1 & 1
\end{array}\right)
$$

are positive definite because

$$
\lambda_{m}(A)=0,9 \text { and } \lambda_{M}(B)=1,1
$$

For such a choice of the matrix-valued function (4), we have

$$
\Theta=\left(\begin{array}{cc}
3,75 & 3,35 \\
3,35 & -3,75
\end{array}\right), \quad \Lambda=\left(\begin{array}{cc}
-0,917 & 0,502 \\
0,502 & -0,917
\end{array}\right)
$$

It is easy to check that matrices $\Theta$ and $\Lambda$ are negative definite. Therefore, all conditions of Theorem 1 are satisfied and the zero solution of system (1) specified by vectors and matrices (18) is absolutely stable on $\mathcal{G}_{s}$.

\section{References}

[1] Djordjevic, M.Z., Stability analysis of large-scale systems whose subsystems may be unstable, Large Scale Systems 5 (1983), 255-262.

[2] Grujić, Lj.T., Martynyuk, A.A. and Ribbens-Pavella, M., Large Scale Systems Stability under Structural and Singular Perturbations, Springer-Verlag, Berlin 1987.

[3] Hahn, W., Stability of Motions, Springer-Verlag, New York 1967.

[4] LaSalle, J.P. and Lefschetz, S., Stability by Lyapunov's Direct Method with Applications, Academic Press, New York 1966. 
[5] Lurie, A.I., Some Nonlinear Problems in Automatic Control Theory, Gostechizdat, Moscow 1951.

[6] Martynyuk, A.A. and Miladzhanov, V.G., Stability theory for large-scale systems with structural perturbation, Electronic Modelling 6 (1991), 10-14.

[7] Martynyuk, A.A. and Stavroulakis, I.P., Direct Lyapunov's matrix-valued functions methods for impulsive systems under structural perturbation, (to appear).

[8] Martynyuk, A.A. and Stavroulakis, I.P., Stability analysis of linear impulsive differential systems under structural perturbation, Ukrain. Math. J., (to appear).

[9] Miladzhanov, V.G., Stability of large-scale impulsive systems under structural perturbations, Dokl. Akad. Nauk Ukraine 12 (1992), 49-52.

\section{Appendix 1}

In order to describe the structurally variable, large-scale system (1) let the following notation be introduced. The structural parameters $S_{i j}:[0, \infty) \rightarrow\{0,1\}$ are binary valued functions of $t$, or $s_{i j}:[0, \infty) \rightarrow[0,1]$, and represent the $(i, j)$-th element of the structural matrix $S_{i}$ of the $i$-th interconnected subsystem $S_{i}$,

$$
S_{i}=\left[s_{i 1} I_{i}, s_{i 2} I_{i}, \ldots, s_{i n} I_{i}\right], \quad I_{i}=\operatorname{diag}\{1,1, \ldots, 1\} \in R^{n_{i}} \times R^{n_{i}}
$$

Notice that it may be, but need not be, required that $s_{i j}(t)=1$ implies $s_{i k}(t)=0$ for all $k \neq j$.

Let

$$
S=\operatorname{diag}\left[S_{1}, S_{2}, \ldots, S_{s}\right], \quad 0_{i j} \in R^{n_{i} \times n_{j}}, \quad i \neq j
$$

Matrix $S(t)$ describes all structural variations of system (1) and will be called the structural matrix of system (1). The set of all possible $S(t)$ will be denoted by $\mathcal{G}_{s}$ and referred to as the structural set of system (1):

$$
\mathcal{G}_{s}=\left\{S: S=\operatorname{diag}\left[S_{1}, S_{2}, \ldots, S_{s}\right], S_{i}=\left[s_{i 1} I_{i}, s_{i 2} I_{i}, \ldots, s_{i n} I_{i}\right]\right\}, i j \in\{0,1\} .
$$

For a detailed discussion of this notion, see [2] and references in this monograph. 


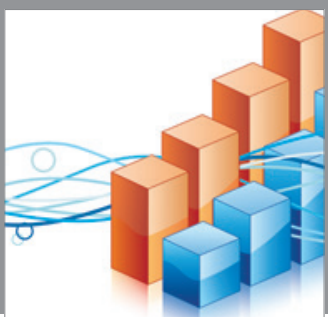

Advances in

Operations Research

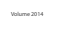

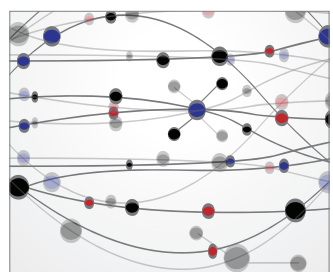

\section{The Scientific} World Journal
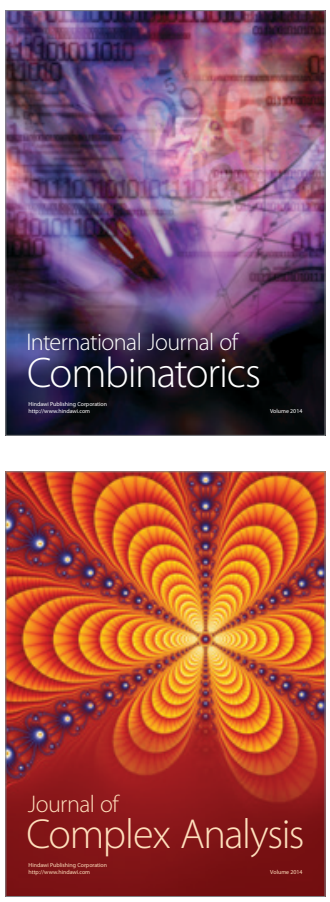

International Journal of

Mathematics and

Mathematical

Sciences
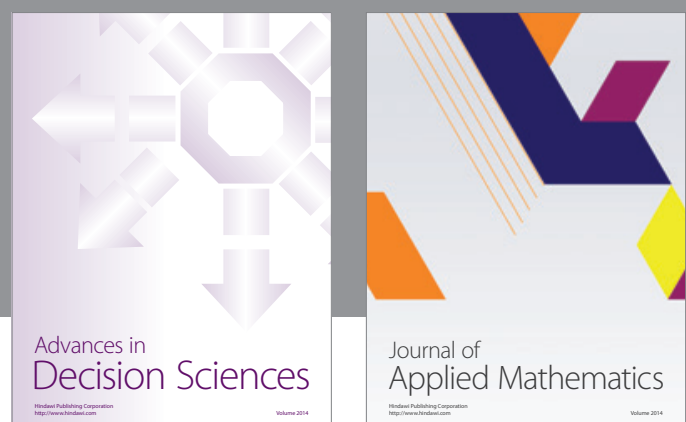

Journal of

Applied Mathematics
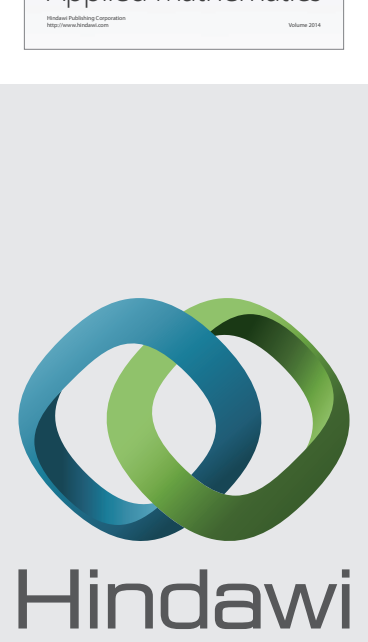

Submit your manuscripts at http://www.hindawi.com
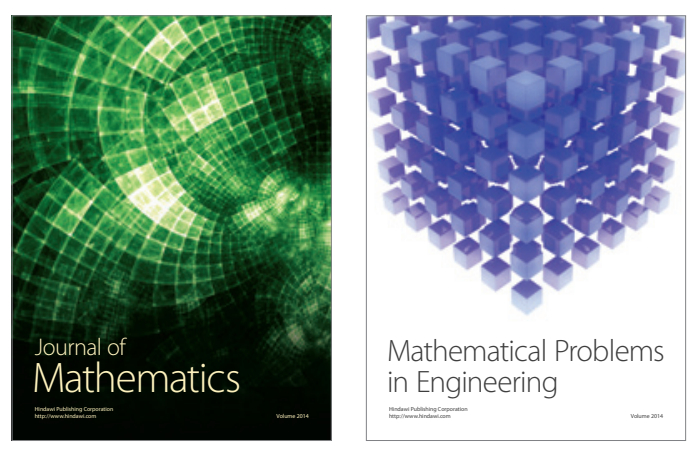

Mathematical Problems in Engineering
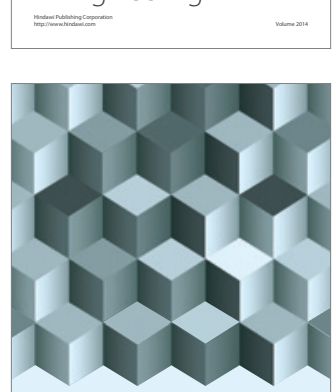

Journal of

Function Spaces
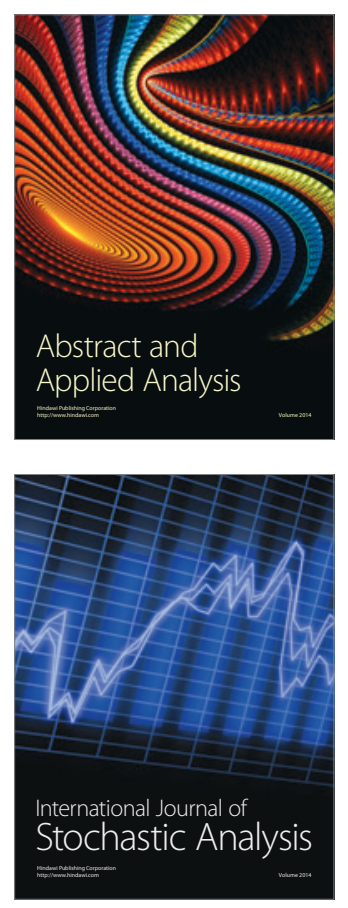

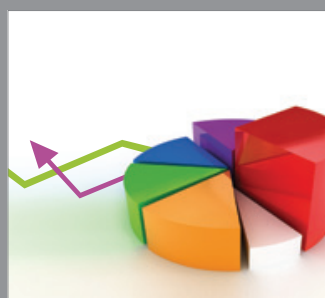

ournal of

Probability and Statistics

Promensencen
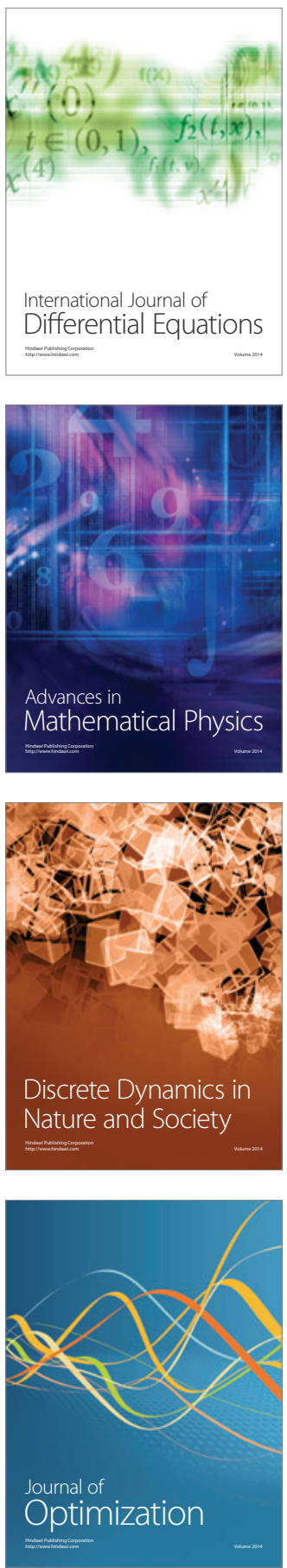